\title{
Cuidados da equipe de enfermagem ao paciente em pós-operatório de bypass gástrico em y de roux
}

\author{
Cares of the nursing team to the post-operative patient of gastric bypass in and of roux \\ Cuidados del equipo de enfermería al paciente en postoperatorio de bypass gástrico en y \\ de roux
}

Cleide Solange Gonçalves ${ }^{1 *}$, Débora Rodrigues de Carvalho ${ }^{1}$, Gabrielle Cristiane de Souza ${ }^{1}$, Lídia Gabrielle Zanola ${ }^{1}$, Luciene Aparecida dos Santos ${ }^{1}$, Marcio Antônio Resende ${ }^{2}$, Gilberto de Souza ${ }^{2}$.

\section{RESUMO}

Objetivo: O presente estudo tem por finalidade descrever quais os cuidados que a equipe de enfermagem deve ter em relação ao pós-operatório de Bypass Gástrico em $Y$ de Roux. Métodos: A metodologia empregada foi à pesquisa bibliográfica nas seguintes fontes Scientific Eletronic Library Online (SCIELO), Literatura Latino-Americana e do Caribe em Ciências de Saúde (LILACS) e base de dados de enfermagem (BDENF). Resultados: O bypass é uma cirurgia invasiva onde o médico acaba retirando uma grande parte do estomago ligando o início do intestino à pequena porção restante do estomago, o que faz com que ocorra diminuição do espaço disponível para a comida, consequentemente reduz a quantidade de calorias absorvidas. Considerações finais: A equipe de enfermagem pode atuar na pós-cirurgia bariátrica junto ao paciente de diversas maneiras, entre elas, ajudando-o na deambulação, na promoção do autocuidado e do medicamento e também as recomendações alimentares que são fundamentais nesta nova fase de vida do paciente pós-cirurgia bariátrica.

Palavras-chave: Cirurgia Bariátrica, Equipe de Enfermagem, Tratamento da Obesidade.

\begin{abstract}
Objective: The purpose of this study is to describe the nursing team's care in relation to postoperative Rouxen-Y gastric bypass. Methods: The methodology used was the bibliographical research in the following sources: Scientific Eletronic Library Online (SCIELO), Latin American and Caribbean Literature in Health Sciences (LILACS) and nursing database (BDENF). Results: The bypass is an invasive surgery where the doctor ends up removing a large part of the stomach connecting the beginning of the intestine to the small remaining portion of the stomach, which causes a decrease in the space available for the food, consequently, reduces the amount of calories absorbed. Final considerations: The nursing team can act in the postoperative bariatric surgery with the patient in a variety of ways, including assisting them in ambulation, promoting self-care and medicine, as well as the dietary recommendations that are fundamental in this new phase of life. patient after bariatric surgery.
\end{abstract}

Words-key: Bariatric Surgery, Nursing team, Treatment of Obesity.

\footnotetext{
${ }^{1}$ Discente do Curso de Enfermagem - Centro Universitário "Presidente Tancredo de Almeida Neves", São João Del Rei - MG. * E-mail: sol-minerinha@hotmail.com

'Docente do Curso de Enfermagem - Centro Universitário "Presidente Tancredo de Almeida Neves", São João Del Rei - MG.
} 


\section{RESUMEN}

Objetivo: El presente estudio tiene por finalidad describir cuáles son los cuidados que el equipo de enfermería debe tener en relación al postoperatorio de Bypass Gástrico en $Y$ de Roux. Métodos: La metodología empleada fue a la investigación bibliográfica en las siguientes fuentes Scientific Eletronic Library Online (SCIELO), Literatura Latinoamericana y del Caribe en Ciencias de la Salud (LILACS) y base de datos de enfermería (BDENF). Resultados: El bypass es una cirugía invasiva donde el médico acaba retirando una gran parte del estómago conectando el inicio del intestino a la pequeña porción restante del estómago, lo que hace que ocurra disminución del espacio disponible para la comida, consecuentemente reduce la cantidad de calorías absorbida. Consideraciones finales: El equipo de enfermería puede actuar en la post-cirugía bariátrica junto al paciente de diversas maneras, entre ellas, ayudándolo en la deambulación, en la promoción del autocuidado y del medicamento y también las recomendaciones alimentarias que son fundamentales en esta nueva fase de vida del paciente, paciente post-cirugía bariátrica.

Palabras clave: Cirugía Bariátrica, Equipo de Enfermería, Tratamiento de la Obesidad.

\section{INTRODUÇÃO}

A obesidade é uma doença crônica, cuja principal característica é o acúmulo de gordura corporal. Tem relação com o aumento do índice de mortalidade e vem acompanhada de algumas comorbidades, como: hipertensão arterial, diabetes mellito, dislipidemias, câncer e doença cardiovascular. (CARVALHO MCVS e LUZ MT, 2013). Em vários estudos de investigação associaram tais doenças com a existência de sobrepeso e obesidade. Duas relevantes investigações selecionaram as mais notáveis pesquisas e salientaram o risco para cada doença (DONINI LM et al, 2012).

Um projeto presumível fundado nas informações epidemiológicos nas ultimas décadas nos EUA, verificou que os indicadores de redução de $1 \%$ no IMC ao longo do tempo seriam possíveis de diminuir cerca de 2,1 milhões de casos incidentes de diabetes, 1,4 milhões de eventos cardiovasculares e 73.000 casos de câncer (MCGEE DL, 2005).

A obesidade já é uma realidade para 18,9\% dos brasileiros. Já o sobrepeso atinge mais da metade da população (54\%). Entre os jovens, a obesidade aumentou 110\% entre 2007 e 2017. Esse índice foi quase o dobro da média nas demais faixas etárias $(60 \%)$. O crescimento foi menor nas faixas de 45 a 54 anos (45\%), 55 a 64 anos (26\%) e acima de 65 anos (26\%). A obesidade é um caso de saúde pública. A crescente incidência nos últimos anos, atingiu pessoas em todo o mundo, e nessa perspectiva, vem desenvolvendo doenças de alto índice de gravidade, podendo muita das vezes ser levada a morte, tanto nos países desenvolvidos como nos em desenvolvimento. Nos últimos anos, houve um aumento da incidência da obesidade em países desenvolvidos e em desenvolvimento. Esse fato, somado aos riscos associados, tais como os distúrbios cardiovasculares, ortopédicos, digestivos, endócrinos, dermatológicos e respiratórios, demonstra que a obesidade é um relevante problema de saúde pública (CARVALHO MCVS e LUZ MT, 2013).

A cirurgia bariátrica é considerada uma das opções para o tratamento da obesidade, sendo esta um procedimento invasivo que é utilizado para diminuir a quantidade de alimento que é absorvido no trato gastrointestinal, tendo como principais benefícios para o paciente à perda do peso, que resulta na cura ou controle das comorbidades, levando a melhora da qualidade de vida, com redução média entre 35 a $40 \%$ (CARVALHO MCVS e LUZ MT, 2013). A cirurgia bariátrica é destinada aos portadores da obesidade mórbida e de acordo com esse procedimento, o índice de reversão é quase nulo, podendo ser considerado muito eficaz. É destinada aos portadores de obesidade mórbida. De acordo com esse procedimento, o índice de reversão é quase nulo, podendo ser considerado muito eficaz. Existe mais de um tipo de cirurgia 
bariátrica, entre as mais conhecidas cita-se a que reduz o tamanho do estomago, que apresenta três variações denominadas: banda vertical ajustável, gastroplastia vertical e gastroplastia vertical com by-pass em y de Roux. A by-pass em y de Roux é também conhecida como Capella ou Fobi-Capella, proporcionando a redução do volume do estômago, ocorrendo uma pequena disabsorção dos alimentos, pois eles deixam de passar pela primeira parte do intestino delgado (CARVALHO MCVS e LUZ MT, 2013).

Este estudo se justifica, principalmente, para que o profissional de enfermagem conheça as propostas cirúrgicas, as vantagens e desvantagens da técnica empregada e o desencadear de cada uma delas, uma vez que essas informações auxiliam os cuidados de enfermagem, que são fundamentais na recuperação pós-operatória do paciente.

Para compreendermos o cuidado da enfermagem, na perspectiva dos profissionais de enfermagem, às pessoas que se submetem à cirurgia de redução de peso, defendemos que este cuidado tem uma dimensão não só técnica, mas também social e cultural. O cuidado à saúde pode ser ofertado no sistema formal de saúde, são os cuidados profissionais, e sua ênfase é sobre os aspectos biológicos e funcionais, mas também pode advir de ações terapêuticas realizadas por famílias e pessoas fora do âmbito profissional, considerados cuidados informais.

\section{METODOLOGIA}

A metodologia empregada foi à pesquisa bibliográfica e como estratégia de busca os achados referentes aos estudos foram obtidos por meio de consulta em três bases de dados: Scientifi Eletronic Library Online (SCIELO), Literatura Latino-Americana e do Caribe em Ciências de Saúde (LILACS) e base de dados de enfermagem (BDENF). O período de publicação dos periódicos ficou estabelecido entre os anos de 2006 a 2017.

\section{RESULTADOS E DISCUSSÃO}

\section{Obesidade}

A obesidade é entendida pelo excesso intenso de gordura corporal no indivíduo. Conforme o Ministério da Saúde, a obesidade traz grandes riscos para a saúde da pessoa, em razão de sua relação com diversas complicações metabólicas. Pode ser entendida como um dano de propriedade multifatorial, pois, suas origens estão associadas a problemas biológicos, históricos, ecológicos, econômicos, sociais, culturais e políticas (CARVALHO MCVS e LUZ MT, 2013).

O Índice de Massa Corporal (IMC) é o critério utilizado em todo o mundo para classificar os indivíduos em peso baixo, peso normal, excesso de peso, obesidade graus I, II ou III, ou seja, é empregado na identificação da condição nutricional das classes populacionais. No entanto, até este momento, não existe um procedimento ordenado da situação nutricional em crianças e adolescentes integralmente válido. $O$ cálculo do IMC se dispõe de maneira que, se divide o peso do paciente pela sua altura elevada ao quadrado (ZIGER M, et al., 2016). Deluchi (2013) relata que existe uma relação tênue entre o comer compulsivo e os gatilhos externos, pois o alimento neste caso acaba servindo como um instrumento de enfrentamento de conflitos interpessoais e situações de estresse.

Para que o indivíduo consiga realizar a cirurgia bariátrica para $\circ$ tratamento da obesidade e consequentemente das suas comorbidades, o mesmo deve estar enquadrado no grau de obesidade III, deve ser avaliado por médicos especializados e ter alguns critérios a serem seguidos, como: IMC acima de $40 \mathrm{~kg} / \mathrm{m}^{2}$ ou acima de $35 \mathrm{~kg} / \mathrm{m}^{2} \mathrm{com}$ comorbidades graves envolvidas, além de não ter obtido sucesso com técnicas não cirúrgicas (STEYER NH et al., 2016).

\section{Cirurgia Bariátrica no Tratamento da Obesidade: Bypass Gástrico em Y de Roux (Rygb)}

Para obesos mórbidos, a cirurgia bariátrica é considerada um tratamento ímpar, pois, traz um resultado considerável e duradouro acerca da perda de peso, e consequentemente uma convalescença das co- 
morbidades relacionadas ao acúmulo de peso. E por essa razão deve ser entendida como um procedimento bastante positivo para a diminuição de peso e continuidade dessa perda em enfermos com obesidade grave. Entretanto, esse tratamento deverá ser feito somente após o paciente já tiver sido submetido a todos os procedimentos tradicionais, e sem os resultados satisfatórios no que se refere à perda de peso, sendo assim, a cirurgia pode ser considerada somente após se descartar os tratamentos convencionais. Donnell (2004).

O tratamento é indicado principalmente para pessoas que venham a apresentar IMC entre $35-40 \mathrm{~kg} / \mathrm{m}^{2} \mathrm{e}$ consequentemente que apresentem alguma comorbidade, sendo necessário que estejam bem informados em relação às mudanças no estilo de vida necessárias após a cirurgia. Os resultados positivos que se obtém nos primeiros anos após a cirurgia devem ser considerados fatores de estímulos necessários para que os pacientes mudem seus hábitos de vida e alimentação. É necessário a prática de atividade física e se também acompanhamento psicológico (BARROS LM et al., 2015).

De acordo com Nora (2016), a cirurgia bariátrica é classificada como restritiva, segundo o mecanismo de ação no qual leva à perda de peso. As cirurgias restritivas acabam limitando a ingestão alimentar por meio da formação de uma pequena bolsa gástrica (gastroplastia, banda gástrica ajustável e gastrectomia vertical), ao contrário dos procedimentos mal absortivos, como no caso do bypass jejuno ileal, que acabam limitando a absorção de nutrientes. Essa característica fez com que a técnica fosse substituída por métodos do tipo misto, ou seja, combinando o efeito restritivo com a má absorção de micronutrientes (bypass gástrico proximal em Y de Roux) e/ou macronutrientes (bypass gástrico distal e desvio biliopancreático).

A escolha pela cirurgia bariátrica no tratamento da obesidade deve ser analisada com muita cautela, pois para alguns pacientes após a cirurgia podem ocorrer deficiências nutricionais, como a carência de vitaminas do complexo B, ferro, ácido fólico, cálcio, zinco, vitamina D e albumina (ZIGER M, et al., 2016).

As cirurgias bariátricas mais utilizadas são o bypass gástrico e a gastrectomia vertical. $O$ bypass gástrico proximal acaba criando uma pequena bolsa gástrica e que não incluiu o fundo do estômago (componente restritivo), uma anastomose gastrojejunal em $Y$ de Roux, de forma a fazer bypass do estômago excluso, duodeno e intestino delgado proximal e em criar um canal biliopancreático de $50 \mathrm{~cm}$ e um canal alimentar de $150 \mathrm{~cm}$. Tal procedimento demonstrou grandes benefícios no metabolismo dos pacientes, principalmente no tratamento das comorbidades, como a Diabetes Mellitus tipo 2, controle glicêmico ou remissão clínica da doença em até $85 \%$ dos casos, levando a uma perda de peso significante, o que o torna um método cirúrgico muito equilibrado, principalmente pela alta taxa de sucesso e pequena taxa de efeitos adversos e de complicações (NORA C, 2016).

De acordo com Acquafresca et al., (2015), entre todos os procedimentos cirúrgicos o by-pass gástrico em $Y$ de Roux (RYGB) é o mais utilizado pelos médicos, pois como pertence ao grupo dos procedimentos combinados, no qual acaba gerando a restrição e má-absorção. A restrição ocorre pela exérese do estomago proximal, o que reduz o seu volume, criando uma bolsa de cerca de 10 a $25 \mathrm{ml}$, o que consequentemente deixa o resto do estômago excluído. A má absorção ocorre devido à divisão do intestino delgado que forma uma alça alimentar (alça de Roux) e outra biliopancreática. A alça alimentar de Roux em Y é criada através da divisão do jejuno $50 \mathrm{~cm}$ abaixo do ligamento duodeno jejunal. Em seguida ela é medida e uma jejunostomia mecânica latero-lateral é criada a $150 \mathrm{~cm}$ abaixo da gastrojejunoanastomose.

Os resultados esperados com a cirurgia bariátrica incluem: perda de peso, melhora das comorbidades relacionadas e da qualidade de vida. No estudo SOS (Swedish Obese Subjects), foi encontrada uma melhora da qualidade de vida, dos parâmetros cardiorrespiratórios (dor torácica, dispneia, apneia do sono e hipertensão) e metabólicos (diabetes e distúrbios lipídicos) associados a uma perda substancial de peso induzida pela cirurgia. A mortalidade perioperatória está em torno de 0,3 a $1,6 \%$ (KARLSSON J SJOSTROM L, SULLIVAN M, 1998).

\section{Cuidados de Enfermagem no Pós-Operatório da Cirurgia Bariátrica}

A cirurgia bariátrica se tornou uma das alternativas no tratamento da obesidade e consequentemente na 
resolução de diversas comorbidades. A assistência de enfermagem abrange todo o perioperatório, inclusive o pós-operatório, contribuindo de maneira significativa para os resultados positivos da cirurgia. No processo de seleção o enfermeiro pode elucidar as dúvidas do paciente e de seus familiares, explicando os testes necessários de diagnóstico e sua importância, além de fazer um histórico de enfermagem completo, com dados relevantes sobre o paciente, explicando as mudanças que a cirurgia bariátrica promove, como perda de peso, alteração nos padrões alimentares, na imagem corporal e na percepção do outro (FERREIRA MBG e FELIX MMS, 2014).

Do ponto de vista psicológico o paciente possui muitas dúvidas e conflitos antes do procedimento cirúrgico e os sintomas mais evidentes são insegurança, medo e ansiedade. Neste momento a equipe de enfermagem é imprescindível, pois ajudará tanto o paciente como a sua família a compreender melhor a cirurgia e prepará-los para a mesma (EDUARDO CA, et al., 2017).

Uma das etapas do Processo de Enfermagem é o Diagnóstico de Enfermagem (DE), que é o meio por onde se faz o julgamento clínico das respostas do paciente aos processos vitais ou em relação aos problemas de saúde atuais. Tal procedimento da assistência acaba permitindo que o enfermeiro venha a conhecer as respostas humanas alteradas no paciente, além de verificar os riscos decorrentes destas alterações, contribuindo para o planejamento de intervenções individualizadas (SANTOS BN, et al., 2015).

De acordo com Moreira (2013), existem alguns cuidados especiais que devem ser levados em consideração pela equipe de enfermagem, referente ao pós-operatório de pacientes que se submeteram à cirurgia bariátrica, uma vez que as comorbidades associadas à obesidade fazem destes pacientes candidatos a alto risco cirúrgico, demandando a identificação correta e oportuna dos problemas pelo enfermeiro, afim de que medidas necessárias para sua solução sejam estabelecidas precocemente.

Uma das complicações comumente encontradas no pós-operatório da bariátrica são a infecção da ferida cirúrgica, que acontece por causa do suprimento inadequado de tecido adiposo e outros fatores que acabam contribuindo para a infecção, como acúmulo de bactéria nas dobras cutâneas próximas a ferida e deiscência da sutura ou excesso de umidade da pele. Outros riscos de complicações que o paciente pode apresentar referem-se à imobilização no pós-operatório, como trombo embolismo pulmonar (TEP), integridade cutânea prejudicada (úlcera de pressão), trombose venosa profunda (TVP) e insuficiência respiratória (NIBI FA e OSTI C, 2014).

O enfermeiro deve estar atento no pós-operatório da cirurgia bariátrica, nas complicações pulmonares, como a pneumonia, infecção traqueobrônquica, atelectasia, ventilação mecânica e/ou intubação orotraqueal prolongadas, insuficiência respiratória aguda e bronco espasmo. A equipe multidisciplinar que atua no perioperatório dos pacientes que se submetem a cirurgia bariátrica deve ter como objetivo prevenir e tratar possíveis complicações oferecendo apoio e cuidado, que deve ser iniciado antes da cirurgia bariátrica e se estender por um mínimo de cinco anos pós-operatório (FERREIRA MBG e FELIX MMS, 2014).

Conforme Ferreira e Felix (2014) os cuidados da equipe de enfermagem em relação ao paciente póscirurgia bariátrica refere-se à promoção da mobilidade no leito e à sua deambulação, minimizando riscos para infecções, cuidando dos curativos, cateteres e drenos, ausculta pulmonar e exercícios respiratórios, administração de analgésicos e antieméticos, antibióticos, monitorização, possíveis complicações, cateterização vesical de demora, orientações quanto ao procedimento cirúrgico, promoção de sono e repouso, medidas de autocuidado e cuidados em casa (ferida operatória e pele), além de visitas de acompanhamento, adoção de protocolo clínico, orientações quanto ao processo cirúrgico, assinatura do termo de consentimento informado na avaliação pré-operatória, instruções quanto à dieta, alterações medicamentosas e sinais de complicações no pós-operatório (FERREIRA MBG e FELIX MMS, 2014).

Segundo Rocha, Lima e Lima (2014), cerca de 0,6 a 7,3\% dos pacientes em pós-operatório precisam de ventilação mecânica, onde entra o papel do enfermeiro para que consiga manter o controle dos sinais vitais, observando o nível de consciência e saturação de oxigênio, incentivando a mudança de decúbito. Diarreias e vômitos são comuns após a cirurgia, pois os pacientes não podem ingerir grande quantidade de líquidos. A partir disso, a equipe de enfermagem deve estimular a ingestão de menores quantidades de alimento e 
em número maior de vezes, verificando o débito urinário, monitorando a presença de íleo paralítico e estimulando a deambulação precoce, garantindo o sucesso da terapêutica.

Outras informações que a equipe de enfermagem deve repassar aos pacientes que se submeteram a cirurgia bariátrica estão relacionadas à alimentação, autocuidado e atividade física. Tais informações servirão de auxílio na adaptação ao novo estilo de vida do paciente. As orientações mais relevantes são a não ingestão de líquidos 15 minutos antes de cada refeição e até 90 minutos, para que se evite a distensão gástrica, estimulando o paciente a seguir a dieta proposta, orientando-o sobre a perda de peso, que será mais eficiente com a prática de exercícios físicos, orientando-o sobre os cuidados com a ferida operatória, evitando o risco de infecções no local, estimulando consequentemente com que a família se torne participativa em todos os cuidados e ações nessa nova etapa da vida do cliente (ROCHA KR, et al, 2014).

O profissional da enfermagem que acompanha o paciente obeso dentro do hospital tem um papel fundamental na interação da equipe. Deve ter um conhecimento tanto prático quanto teórico acerca do tratamento oferecido. Sempre está ciente de todos os processos pré, trans e pós-operatório, podendo assim, proporcionar uma assistência de qualidade procurando sempre alcançar um resultado mais satisfatório possível (ROCHA KR, et al, 2014).

\section{CONSIDERAÇÕES FINAIS}

Os pacientes submetidos à cirurgia bariátrica necessitam de cuidados de Enfermagem diferenciados no perioperatório. Os cuidados de Enfermagem identificados na presente revisão englobam principalmente aspectos referentes à mobilidade e deambulação, profilaxia tromboembolística, terapia medicamentosa, cuidados com drenos, curativos e cateteres, educação do paciente no pré e pós-operatório e ações individualizadas que atendam as reais necessidades dos pacientes. A conduta do enfermeiro precisa estar condizente com a promoção da saúde e da prevenção dos danos e agravos e suas ações devem estar voltadas para solucionar problemas, o que pode ocorrer mediante a uma assistência integral.

O enfermeiro, participante em todo o processo, necessita de aprimoramento constante, sendo mediador entre cada profissional da equipe, atento inclusive às demandas dos familiares, esclarecendo dúvidas e transmitindo segurança. Contudo, evidencia-se que os profissionais dão enfoque ao biológico em detrimento dos aspectos psicossociais comprometendo a qualidade de vida e os resultados do tratamento. Sendo assim, cabe ao enfermeiro refletir sobre a assistência prestada, levando em consideração a pluralidade inerente a esse paciente.

\section{REFERÊNCIAS}

1. ACQUAFRESCA PA. Complicações cirúrgicas precoces após by-pass gástrico: revisão da literatura. $A B C D$ Arq Bras Cir Diag. 2015; 28(1): 74-80.

2. Associação Brasileira para o Estudo da Obesidade e da Síndrome Metabólica (Abeso). Diretrizes Brasileiras de Obesidade. 3a Ed. 2009.

3. BAGATINI A, et al. Anestesia para cirurgia bariátrica. Avaliação retrospectiva e revisão da literatura Rev Bras Anestesiol. Vol. 56. Num. 3. 2006. p. 205-222.

4. BARROS LM, FROTA NM, MOREIRA RAN, et al. Avaliação dos resultados da cirurgia bariátrica. Rev Gaúcha Enferm. 2015; 36(1): 21-27.

5. BRUNNER e SUDDARTH, Tratado de enfermagem médico-cirúrgica / [editores] Suzanne C. Smeltzer...et al.]; [revisão técnica Isabel Cristina Fonseca da Cruz, Ivone Evangelista Cabral; tradução Antonio Francisco Dieb Paulo, José Eduardo Ferreira de Figueiredo, Patricia Lydie Voeux]. - Rio de Janeiro: Guanabara Koogan, 2011. 2v.: il. ; $21 \times 28 \mathrm{~cm}$.

6. CAMPOS JM. Certificado de Área de Atuação em Cirurgia Bariátrica: mais uma conquista da SBCBM. ABCD Express Edit. 2015; 01(01): 6-7.

7. CARVALHO MCVS, LUZ MT. Práticas de saúde, sentidos e significados construídos: instrumentos teóricos para sua interpretação. Interface. 2013; 13(29): 313-26. 
8. DELUCHI M. Terapia cognitivo-comportamental e obesidade. In: Araújo, R. B., Piccoloto, N. M.; Wainer, R. (Orgs.). Desafios clínicos em terapia cognitivo-comportamental. São Paulo: Casa do Psicólogo, 2013.

9. EDUARDO CA, SILVA AA, CRUZ CD, et al. Cirurgia Bariátrica: a percepção do paciente frente ao impacto físico, psicológico e social. Rev Enferm Centro-Oeste Mineiro. 2017; 7 (1173): 1-11.

10. FELIX LG, SOARES MJGO, NOBREGA MML, et al. da. Protocolo de assistência de enfermagem ao paciente em pré e pós-operatório de cirurgia bariátrica. Rev Bras Enferm. 2012; 65(1): 83-91.

11. FERREIRA MBG, FELIX MMS. Cuidados de enfermagem no perioperatório de pacientes submetidos à cirurgia bariátrica. Rev Rene. 2014; 15 (4): 710-9.

12. KARLSSON J, SJOSTROM L, SULLIVAN M. Two-year follow up ofhealth-related quality of life (HRQL) and eating behavior after gastric surgery for severe obesity: Swedish ObeseSubjects (SOS) - an intervention study of obesity. Int Jobes Relat Metab Disord 1998; 22:113-26.

13. LIMA ACR, OLIVEIRA AB. Fatores psicológicos da obesidade e alguns apontamentos sobre a terapia cognitivo-comportamental. Mudanças Psic Saúde. 2016; 24(1): 1-14.

14. MARQUES G. Assistência de enfermagem ao paciente submetido gastroplastia: um estudo bibliográfico. Trabalho de Conclusão de Curso (Curso de Enfermagem), Instituto Municipal de Ensino Superior de Assis/Assis, 42p. 2015.

15. MOREIRA RAN. Diagnósticos de enfermagem, fatores relacionados e de risco no pós-operatório de cirurgia bariátrica. Rev Esc Enfer USP. 2013; 47(1): 168-75.

16. NEGRÃO, Renata de Jesus da Silva. Cirurgia bariátrica: revisão sistemática e cuidados de enfermagem no pós-operatório. 2006. Dissertação (Mestrado em Enfermagem na Saúde do Adulto) - Escola de Enfermagem, Universidade de São Paulo, São Paulo, 2006.

17. NIBI FA, OSTI C. Cuidados intensivos no pós-operatório imediato de cirurgia bariátrica. Rev UNINGÁ. 2014; 39(1): 149-158.

18. NORA C. Gastrectomia vertical e bypass gástrico no tratamento da síndrome metabólica. Rev Port Endo Diab Met. 2016; 11(1): 23-29.

19. ROCHA KR, LIMA ALB, LIMA JR, et al. Assistência de Enfermagem no Pós-Operatório de Pacientes Submetidos á Cirurgia Bariátrica. SEMESP. 2014; 1(1): 1-4.

20. SANTOS BN, NOVELLI MC, FONTES CMB, et al. Diagnósticos de enfermagem em pós-operatório imediato de cirurgia bariátrica em terapia intensiva. Rev Enfer UFPE. 2015; 9(4): 7247-54.

21. SOUSA P, BASTOS AP, VENÂNCIO C, et al. Acta Med Port. 2014; 27(4): 450-457.

22. STEYER NH, OLIVEIRA MC, GOUVÊA MRF, et al. Perfil clínico, diagnósticos e cuidados de enfermagem para pacientes em pós-operatório de cirurgia bariátrica. Rev Gaúcha Enferm. 2016; 37(1): e5017.

23. TAVARES TB, NUNES SM, et al. Mariana Oliveira Obesidade e qualidade de vida: revisão da literatura. Revista Médica de Minas Gerais. Belo Horizonte, v. 20, n. 3, p. 359-366, ago. 2010.

24. ZIGER M, et al., Review: Carbon onions for electrochemical energy storage; J. Mater. Chem. A, 2016. 